\title{
Aging, Cognitive Decline and Hearing Loss: Effects of Auditory Rehabilitation and Training with Hearing Aids and Cochlear Implants on Cognitive Function and Depression among Older Adults
}

\author{
Alessandro Castiglione ${ }^{a} \quad$ Alice Benatti $^{a}$ Carmelita Velardita ${ }^{a} \quad$ Diego Favaro $^{a}$ \\ Elisa Padoan ${ }^{a}$ Daniele Severi $^{\mathrm{a}}$ Michela Pagliaro $^{\mathrm{a}}$ Roberto Bovo ${ }^{\mathrm{a}}$ \\ Antonino Vallesib ${ }^{b}$ Carlo Gabellic Alessandro Martinia, b \\ ${ }^{a}$ Department of Neurosciences and Complex Operative Unit of Otolaryngology, University Hospital of Padua, \\ ${ }^{b}$ Department of Neurosciences and Center for Cognitive Neuroscience, University of Padua, and ${ }^{\mathrm{C}}$ Regional Center \\ for the Study and Treatment of the Aging Brain, Department of Internal Medicine, University of Padua, Padua, Italy
}

\section{Key Words}

Elderly $\cdot$ Cochlear implantation $\cdot$ Hearing aid $\cdot$ Cognitive decline $\cdot$ Hearing loss $\cdot$ Depression

\footnotetext{
Abstract

A growing interest in cognitive effects associated with speech and hearing processes is spreading throughout the scientific community essentially guided by evidence that central and peripheral hearing loss is associated with cognitive decline. For the present research, 125 participants older than 65 years of age (105 with hearing impairment and 20 with normal hearing) were enrolled, divided into 6 groups according to their degree of hearing loss and assessed to determine the effects of the treatment applied. Patients in our research program routinely undergo an extensive audiological and cognitive evaluation protocol providing results from the Digit Span test, Stroop color-word test, Montreal Cognitive Assessment and Geriatric Depression Scale, before and
}

after rehabilitation. Data analysis was performed for a crosssectional and longitudinal study of the outcomes for the different treatment groups. Each group demonstrated improvement after auditory rehabilitation or training on shortand long-term memory tasks, level of depression and cognitive status scores. Auditory rehabilitation by cochlear implants or hearing aids is effective also among older adults (median age of 74 years) with different degrees of hearing loss, and enables positive improvements in terms of social isolation, depression and cognitive performance.

(c) 2016 The Author(s)
Published by S. Karger AG, Basel

\section{Introduction}

The world's population is continuously expanding and is expected to reach 9 billion by 2045 and, concurrently, the population's age distribution shows a constant growth

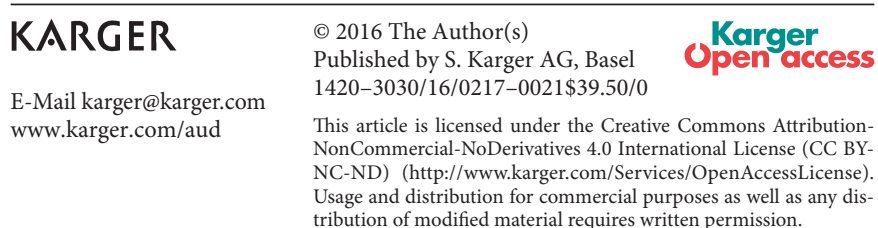

Alessandro Castiglione, $\mathrm{MD}, \mathrm{PhD}$

Department of Neurosciences - Otolaryngology

University Hospital of Padua

2, Giustiniani, IT-35100 Padua (Italy)

E-Mail alessandro.castiglione@ unipd.it 
in the proportion of people over 65 years of age [Van Bavel, 2013]. With increasing age, the prevalence of both hearing loss and dementia increases, exceeding $10 \%$ of individuals beyond the age of 65 years. Clearly, the epidemiological data on hearing-impaired people and patients with cognitive decline are a cause for global and economic concern [Prince et al., 2013].

The continuously expanding clinical indications for the most advanced auditory-function treatment options, including digital hearing aids (HAs) and cochlear implants (CIs), have led to increased expectations for their potential positive effects also on cognitive functions and mood disorders among older adults [Francis et al., 2015; Mosnier et al., 2015; Poissant et al., 2008; Shin et al., 2007]. Several studies have confirmed the correlation between hearing loss and cognitive decline [Lin, 2011; Lin et al., 2011a, b, 2013, 2014], and it has become increasingly evident that even very old people can benefit from procedures, such as cochlear implantation, that previously were recommended mainly for younger hearing-impaired patients [Castiglione et al., 2015; Ciorba et al., 2012]. Growing interest has been spreading in the scientific community for the potential to influence cognitive function, focusing on the possible role of auditory rehabilitation through digital devices in counteracting dementia [Fattahi et al., 2015; Martini et al., 2014; Mosnier et al., 2015]. The restoration of sensory functions among hearing-impaired older adults may allow for significant positive effects in their cognitive status. Auditory rehabilitation can enhance and restore the auditory perceptual skills that are essential for spoken language and, as a consequence, it is theorized that it may also help to ameliorate the central management of other cognitive resources associated with speech and hearing processes. Despite the fact that there is a paucity in conclusive evidence available to further define such theories, several studies have speculated on the potential effects of auditory training on cognitive functions, essentially based on the findings that central and peripheral hearing loss is correlated with cognitive decline [Lin et al., 2013, 2014].

Considering that auditory rehabilitation as a means of providing adequate auditory input can help to reduce the cognitive 'load' caused by hearing loss and, as a consequence, has the potential to reduce the associated physical and psychosocial consequences, the aim of our study was to investigate the effects of restoring hearing ability through CIs and HAs on depression and cognitive functions among older adults affected by varying degrees of sensorineural hearing loss.

\section{Methods}

\section{Study Design}

Via a hospital case file review, hearing-impaired patients of more than 65 years of age, affected with mild to profound hearing loss examined between July 2012 and April 2015, were enrolled in the present study at the ENT Unit, Department of Neurosciences, University Hospital of Padua. Formal, written, informed patient consent was obtained prior to their participation in the study. Data from repeated, longitudinal assessments for intrasubject and cross-sectional intersubject data were gathered and analyzed accordingly.

\section{Subjects}

Of the 125 subjects enrolled in the present research, 105 were hearing-impaired patients and 20 were normal-hearing controls. Due to the varying degrees of hearing loss and the associated auditory rehabilitation needs, the participants were divided into 6 groups according to the hearing device configuration (bilateral or unilateral), device (HA, CI or none) and degree of hearing loss (table 1). Group A consisted of patients with bilateral hearing loss who were candidates for hearing rehabilitation with bilateral HAs (firsttime users). Group B was composed of patients with bilateral hearing loss who were active users of HAs (bilateral, behind-the-ear HAs) and had at least 6 months of auditory training. Group $\mathrm{C}$ were patients who wore a unilateral $\mathrm{HA}$ and had training within the last 2 years with digital device(s). Group D represented patients who were eligible audiologically for auditory rehabilitation with HAs but were without a HA and had never received auditory training (were nonusers for personal reasons, i.e. underestimated the impact of their own hearing deficit). Group E consisted of individuals with profound sensorineural hearing loss who were successful candidates for cochlear implantation with 1 year of CI experience. Group F were control subjects with normal hearing, without additional health complications and within the same age range.

\section{Evaluations}

Routine audiological evaluations including pure-tone and speech audiometry were performed with all patients. Select cases were further screened for cognitive decline and depression through the Montreal Cognitive Assessment (MoCA) [Nasreddine et al., 2005] and the Geriatric Depression Scale (GDS) [Sheikh and Yesavage, 1986], and assessment of short-term memory and executive function were assessed via the Digit Span test (DST) [Leung et al., 2011] and the Stroop color-word test (Stroop test) [Stroop, 1935], respectively. Evaluation schedules and materials were tailored for the individual according to their hearing loss and hearing treatment program and were included for comparison in crosssectional and/or longitudinal study analysis (table 1). Assessments were carried out in the clinic for each subject by an experienced specialist clinical team.

\section{Materials}

The DST is a short-term memory task where the patient's memory is measured by the longest list of numbers they can repeat in correct order immediately after presentation [Leung et al., 2011; Muangpaisan et al., 2010; Schroeder et al., 2012; Sung et al., 2012]. The Stroop test is a measure of reaction time and investigates executive function and selective attention [Mackin et al., 2010; Seo et al., 2008; Rivera et al., 2015]. The Stroop test consists of words with
Castiglione et al. 
Table 1. Stratification of elderly patient cohort to groups A-E according to the degree of hearing loss and auditory rehabilitation received $(\mathrm{n}=125)$

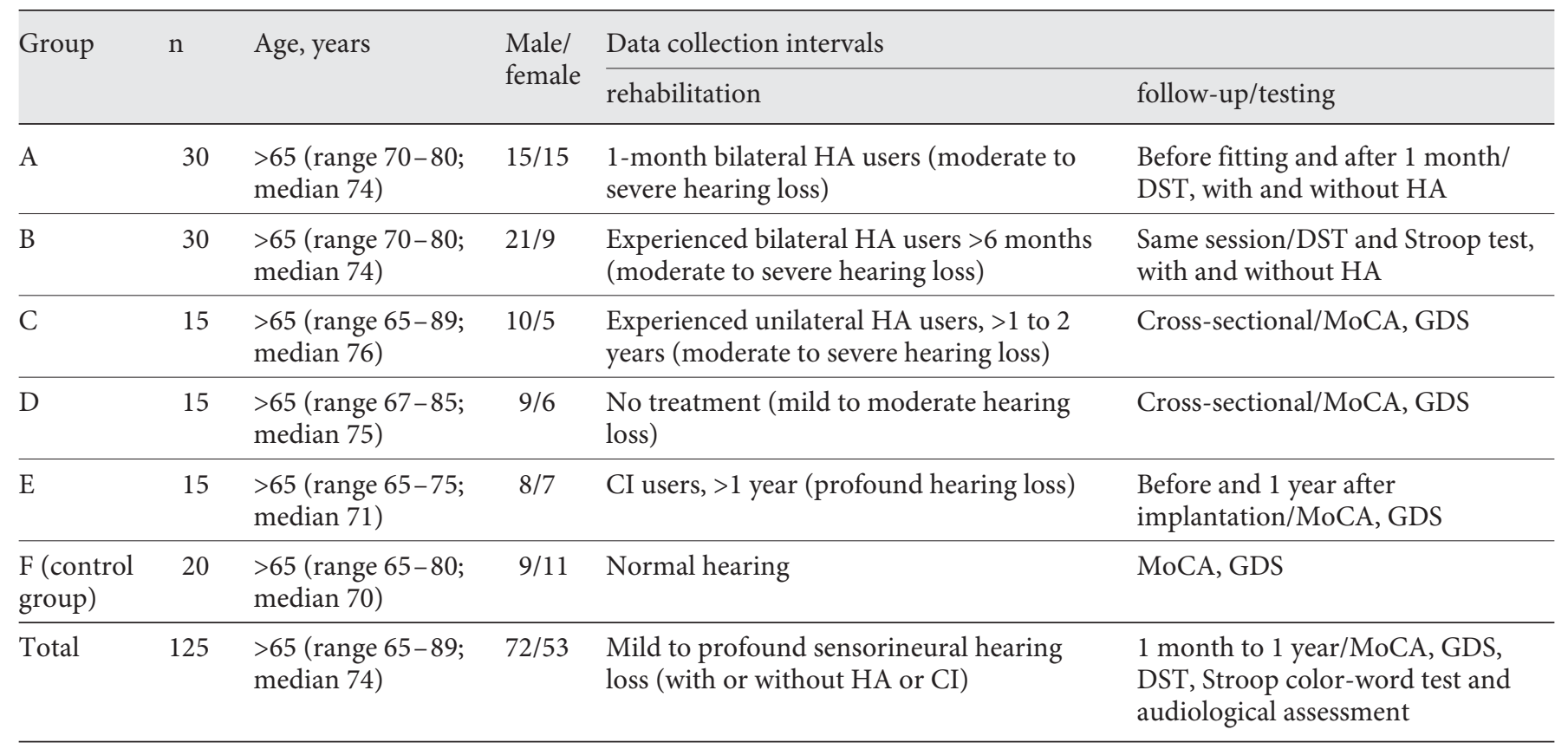

$\mathrm{n}=$ Number of subjects; DST = Digit Span test; MoCA = Montreal Cognitive Assessment; GDS = Geriatric Depression Scale.

different colors that do not match the names of the words (i.e. 3 pages, each with 100 items randomly organized into 5 columns).

The MoCA is a cognitive function screening test of various cognitive domains and was included as it has been shown to have $90 \%$ sensitivity and $87 \%$ specificity that enables identification of $90 \%$ of cases with mild cognitive impairment (MCI) and $100 \%$ of patients with mild Alzheimer disease. A score of 26/30 and higher on the MoCA is considered normal, and results are corrected with coefficients for different levels of education [Nasreddine et al., 2005]. The GDS as a measure of depression was included as it is observed to have a $92 \%$ sensitivity and an $89 \%$ specificity when evaluated against diagnostic criteria. The validity and reliability of the tool have been supported through findings in both clinical and research practice [Lesher and Berryhill, 1994]. Scores on the GDS range between 0 and 30 with $0-9$ as 'normal', $10-19$ as 'mildly depressed' and 20-30 as 'severely depressed' [Lesher and Berryhill, 1994].

Administration of cognitive tests was performed under supervision of the same trained professional. The sequence of testing across subjects was randomized to prevent an order effect. The test setting was kept constant across patients throughout the clinical assessments to reduce systematic errors and to permit comparison of study outcomes, longitudinally and cross-sectionally.

Longitudinal, repeated assessments were performed as described in table 1 for groups A, B and E. In summary: newly fitted bilateral HA users, group A, were tested prior to and following 1 month of auditory training with bilateral behind-the-ear digital HAs on the DST to assess short-term memory. Current/experienced bilateral HA users, group B, were tested acutely in a single session on the DST and the Stroop test in both aided and unaided

Aging - Cognition and Depression conditions to test short-term memory and executive function. The CI users, group E, were assessed before implantation and 1 year after implantation on the MoCA and GDS to assess cognitive function in various domains and the level of depression. In addition, cross-sectional analysis was performed through assessment and comparison of test results for the normal-hearing controls, with group F, long-term unilateral HA users, group C (i.e. trained unilateral HA users), and for hearing-impaired patients receiving no auditory training or device fitting, group D (untrained patients).

\section{Statistical Analysis}

The test results from longitudinal and cross-sectional assessments were analyzed with two-tailed Student's t test for paired and unpaired data and linear regression analysis for correlations among variables such as age, cognitive function, hearing loss and outcomes after auditory rehabilitation. ANCOVA was used to compare regression lines, when appropriate. Statistical difference results from longitudinal and cross-sectional data comparisons were considered significant at $\mathrm{p}$ value $<0.05$.

\section{Results}

The study cohort and subgroup characteristics are presented in table 1 for the stratified treatment groups summarizing age, gender, rehabilitation applied and tests used across the 5 treatment groups for hearing-impaired 


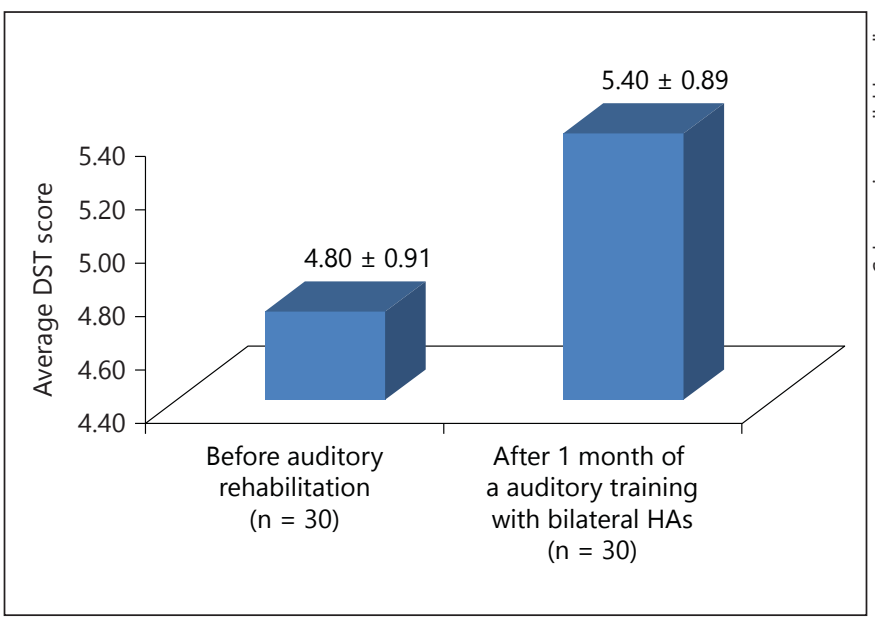

Fig. 1. DST for group A, before and after short-term bilateral HA use (1 month). A significant improvement after auditory rehabilitation is observed $(\mathrm{p}<0.05)$.

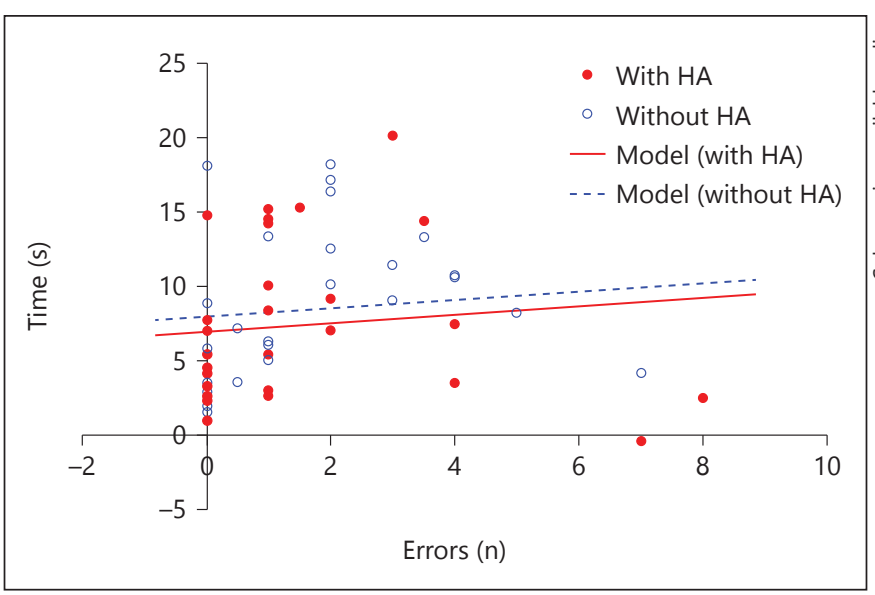

Fig. 2. Stroop test for long-term bilateral HA users, group B, in aided and unaided listening conditions measured in the same session. Scatterplots display results with HA (solid circle) and without HA (open circle) showing the correlation for response time versus errors $\left(R^{2}=0.019\right)$. Regression lines are shown for each listening condition. No statistically significant difference between the listening conditions is observed.

elderly patients (groups A-E) and the normal-hearing control group (group F).

As anticipated, generally, the clinical treatment applied, including the type of device provided and hearing rehabilitation applied was considered audiologically appropriate for the degree and hearing loss configuration

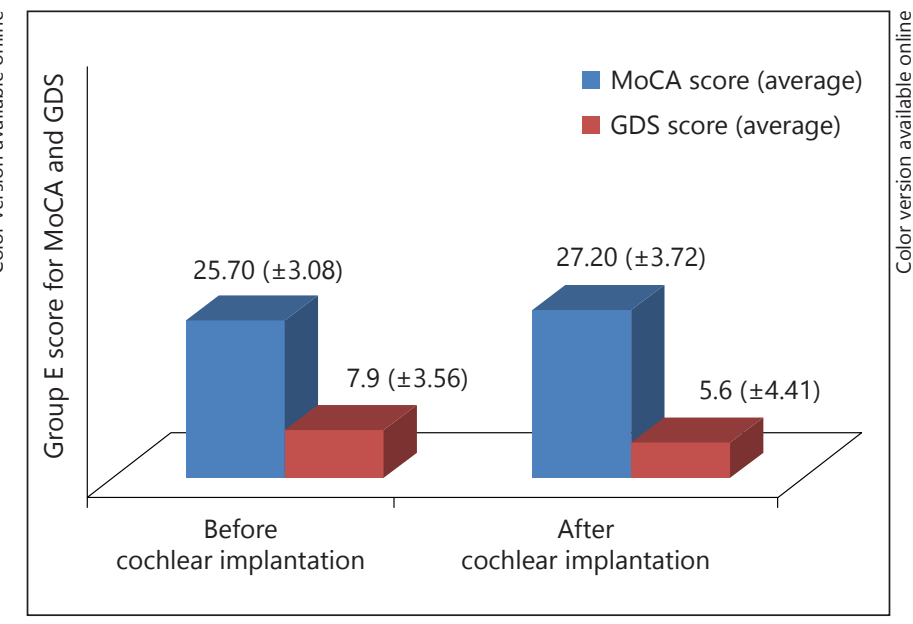

Fig. 3. GDS and MoCA test scores for the implanted patients in group E, before and 1 year after implantation. A statistically significant improvement is observed for each measure after implantation $(\mathrm{p}<0.01)$.

and their individual hearing needs for all subjects. The patient groups are described in detail in table 1.

Group A revealed a significant improvement for the DST (short-term memory test) after 1 month of auditory training with bilateral HAs compared to before auditory rehabilitation (fig. 1). When tested in the same session, group B, the long-term bilateral HA users, also demonstrated a significant improvement on the DST when comparing aided and unaided conditions (not illustrated). Assessment of executive function via the Stroop test for group B revealed no statistically significant difference (fig. 2); however, a trend for better performance with HAs was observed indicating a potential for a reduced number of errors in the aided condition as well as slightly shorter response times relative to the unaided condition.

Among patients who underwent cochlear implantation (group E), the auditory rehabilitation resulted in a positive effect on cognitive performance and reduction in the level of depression after 1 year of implant experience showing a significant effect with $\mathrm{p}<0.01$ (fig. 3). Examination of their patient records confirmed surgeries were uneventful and there were no reports of postsurgery complications for all subjects. Audiologically, on routine clinical measures, implanted group E subjects demonstrated significant improvements in speech recognition tasks and aided thresholds, which has been reported in detail in a previous publication [Castiglione et al., 2015]. As shown in figure 4, linear regression analysis of the results for the implanted group E confirms a mild negative correlation 


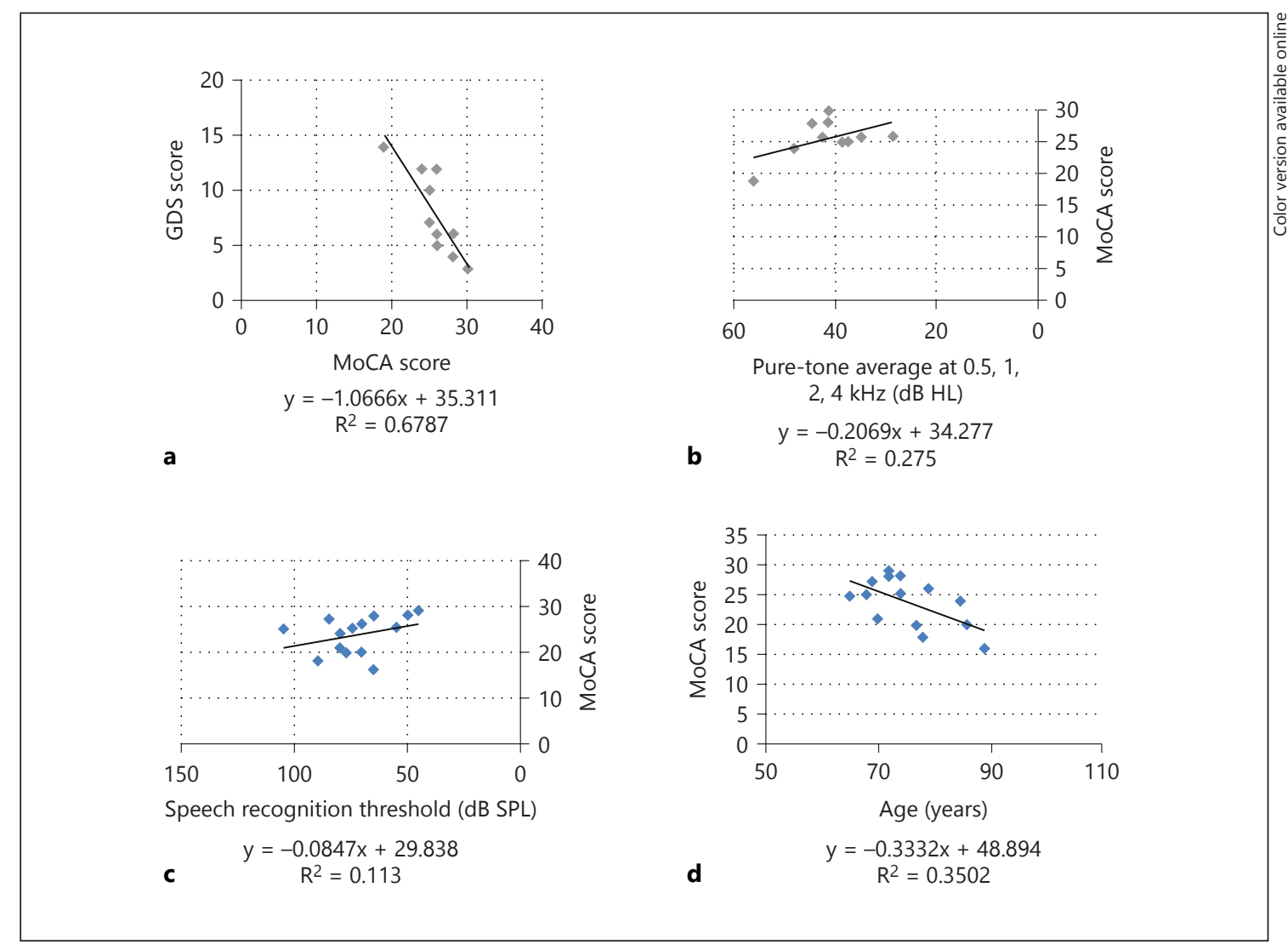

Fig. 4. Correlation of MoCA total score with various determinants for implanted patients in group E 1 year after implantation. a MoCA versus GDS scores, showing a negative correlation. b MoCA versus pure-tone average thresholds, showing a positive correlation. c MoCA versus aided speech reception threshold scores, showing a mild positive correlation. $\mathbf{d}$ MoCA versus age at implantation, showing a negative correlation with increasing age.

between age at implant and cognitive function, and also between assessments of cognitive function and depression with better outcomes on the GDS shown in patients with higher scores on the MoCA. No significant gender effect upon outcomes was observed.

As shown in figure 5, significantly better group outcomes are observed for cognitive function and depression for long-term unilateral HA users in group $\mathrm{C}$, and for the normal-hearing control group F compared to group D who received no treatment, displaying abnormal cognitive function and borderline depression. Comparing postimplantation scores for group E with those displayed for normal-hearing listeners in group $\mathrm{F}$ and for long-term unilateral HA users in group $\mathrm{C}$ suggests no significant differences for outcomes between the groups on the MoCA or GDS.

\section{Discussion}

The effectiveness of cochlear implantation as a treatment for the hearing-impaired elderly, as demonstrated by improved hearing thresholds and speech understanding, has been widely established by several published studies [Bovo et al., 2011a; Chung et al., 2012; Ciorba et al., 2012; Martini et al., 2013]. The data from our study indicates the positive effects of providing effective auditory input with HAs or CIs combined with auditory training beyond hearing function alone.

We demonstrate a positive effect of auditory input upon short-term memory for both groups of short-term and long-term bilateral HA users via improved scores on the DST in the aided listening compared to the unaided listening condition. The effect of auditory input versus no auditory input for executive function as demonstrated via the Stroop test in the same session for group B, while not 


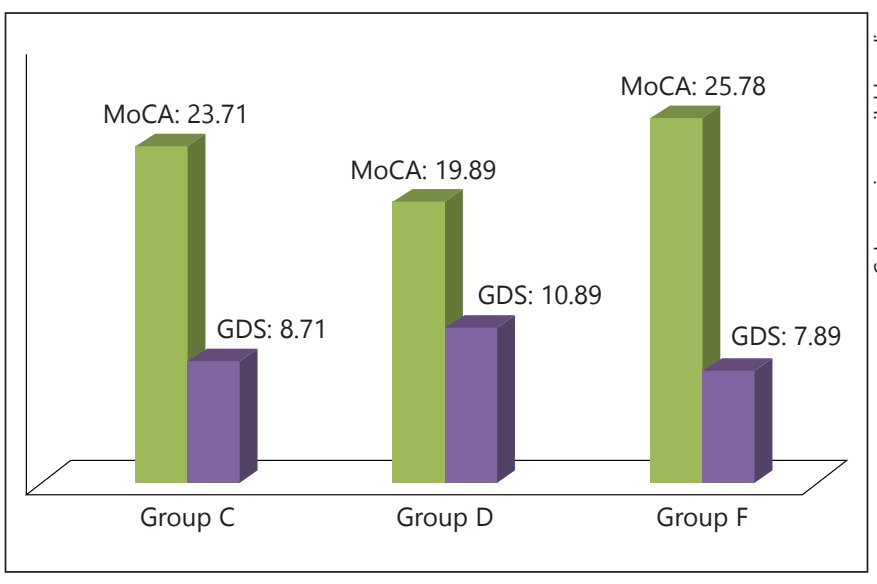

Fig. 5. Average MoCA and GDS scores for 3 groups of patients: group $\mathrm{C}=$ long-term unilateral HA users with moderate to severe hearing loss; group $\mathrm{D}=$ untreated hearing-impaired elderly with mild to moderate hearing loss; group $\mathrm{F}=$ normal-hearing controls. Statistically significant differences for group scores are noted between group $\mathrm{C}$ and group $\mathrm{D}(\mathrm{p}<0.05)$, and between group $\mathrm{F}$ and group $\mathrm{D}(\mathrm{p}<0.05)$. No difference is observed between scores for groups $\mathrm{C}$ and $\mathrm{F}$.

showing a significant difference between the conditions suggests a trend for superior performance in the aided condition and a slightly shorter response time compared to the unaided condition. Nonetheless, subjects were observed to spend more time correcting themselves during the test in the aided condition while aiming to reduce the number of errors. Interestingly, the ANCOVA showed a significant difference at the interception of the y-axis in errors and revealed a mild positive correlation with better performance in the aided condition overall.

The level of depression screened by the use of the GDS, both prior to implant treatment and readministered after implantation, for group E confirmed a significant positive impact of implant treatment upon the level of depression. The MoCA, used as a screening test of cognitive function, is suitable to identify the presence of an MCI before and after treatment of auditory impairment in elderly subjects. As demonstrated by our results, cognitive function improved following implant treatment for subjects in group E compared to their preimplantation status. Furthermore, at the postimplantation interval, CI recipients performed similarly to subjects with an asymmetrical hearing loss using a unilateral HA, group C, and to subjects with normal hearing, group F.

A further examination of pre- and postauditory treatment effects for groups $\mathrm{C}$ and $\mathrm{E}$ via the MoCA was undertaken and compared to that reported in the literature for
Table 2. Average scores obtained at pre- and posttreatment intervals for the cognitive subtask of the MoCA for study groups $\mathrm{C}$ and $\mathrm{E}$

\begin{tabular}{lllllll}
\hline Items/skills & MCI & AD & Normal & $\begin{array}{l}\text { HI- } \\
\text { pre }\end{array}$ & $\begin{array}{l}\text { HI- } \\
\text { post }\end{array}$ & $\begin{array}{l}\text { Diff. } \\
\text { (post/pre) }\end{array}$ \\
\hline Visuospatial/ & & & & & & \\
executive & 3.18 & 2.08 & 4.23 & 3.35 & 3.91 & 0.57 \\
Naming & 2.64 & 2.19 & 2.88 & 2.70 & 2.80 & 0.10 \\
Attention & 5.41 & 3.98 & 5.68 & 5.27 & 5.27 & 0.00 \\
Language & 2.2 & 1.69 & 2.7 & 2.43 & 2.68 & 0.25 \\
Abstraction & 1.43 & 0.99 & 1.83 & 1.40 & 1.55 & 0.15 \\
Memory & 1.17 & 0.52 & 3.73 & 1.45 & 2.51 & 1.07 \\
Orientation & 5.52 & 3.92 & 5.99 & 5.75 & 5.95 & 0.21
\end{tabular}

Scores combined at HI-pre and HI-post treatment intervals. Average data sets for MCI, Alzheimer disease (AD) and normal elderly groups were reported for elderly individuals by Nasreddine et al. [2005]. HI-pre = Hearing-impaired patients before or without auditory rehabilitation; HI-post = hearing-impaired patients with or after auditory rehabilitation; diff. (post/pre) = difference in scores per subsection comparing scores before device use or without auditory rehabilitation to those after device use or with auditory training.

elderly subjects with normal cognitive function, MCI and Alzheimer disease [Nasreddine et al., 2005]. In order to evaluate the contribution of single tasks or items constituting the MoCA, scores are divided into the 7 cognitive subtasks. As shown in table 2, before treatment, generally speaking, performance is more similar to that reported for those with $\mathrm{MCI}$, and after treatment performance approaches that reported for individuals with normal cognitive function. Surprisingly, the improvements for our treatment groups might not directly be derived exclusively from the restoration of their auditory function. The analysis of the subtasks of the MoCA test showed that the greatest increment after auditory treatment and training was observed for long-term memory (average 1.07), followed by tasks assessing visuospatial and logical executive skills (average 0.57 ). Thus, the most significant contribution to the improvement in MoCA scores following auditory treatment is from the positive impact upon long-term memory skills. Notably the average pretreatment scores for long-term memory are comparable to those with MCI reported in the literature [Nasreddine et al., 2005].

As demonstrated for our CI recipients, following longterm use, cognitive function showed a mild positive correlation with routinely measured audiological outcomes for pure-tone and speech audiometry. Conversely, GDS scores were negatively correlated with audiometric outcomes, even though it is difficult to assess and to evaluate 
the real effect of mood disorders among hearing-impaired patients.

Review of the literature led to 6 possible explanations that may further support the findings in our study for elderly patients following treatment of their hearing impairment especially for short-term memory, overall cognitive function and depression. These include the following:(1) reducing social isolation and improving depression symptoms could explain some early effects [Acar et al., 2011; Boi et al., 2012]; (2) electrical stimuli may allow the preservation of the function and 3-dimensional structure of the peripheral and central synapses [Kumar and Foster, 2007; Ryugo et al., 2005; Wong et al., 2009, 2014]; (3) auditory rehabilitation can counteract negative neuroplasticity processes [Lazard et al., 2010, 2011, 2013]; (4) the effect of auditory/speech training may positively influence working memory and learning abilities; (5) improvements in self-motivation, self-esteem or self-confidence after rehabilitative procedures occur resulting in a positive effect on cognitive skills, and (6) a placebo effect.

The hearing system should be considered an important window for investigations into neurodegenerative disorders, and the auditory rehabilitation options currently available should not be denied to elderly patients with cognitive impairment. The finding that short-term memory contributes to word identification and correct recall is confirmed by our current study [Drewnowski and Murdock, 1980; Watkins et al., 1992]. Working memory has a crucial role in understanding spoken words in noisy environments, and as a consequence, assessment of long-term memory function could potentially serve as a predictive factor for rehabilitative outcomes.

Further studies are needed to confirm these preliminary data; however, the present research suggests that early auditory rehabilitation is important even among older adults as it can lead to extended benefits beyond hearing ability including cognitive function and depression [Benatti et al., 2013; Bovo et al., 2011a, b; Ciorba et al., 2012].

\section{Acknowledgments}

A.V. is funded by an ERC Starting Grant, 7th Framework Programme (FP7/2007-2013, GA No. 313692, LEX-MEA).

\section{Disclosure Statement}

The authors state that there is no conflict of interest to be disclosed.

\section{References}

Acar B, Yurekli MF, Babademez MA, Karabulut H, Karasen RM: Effects of hearing aids on cognitive functions and depressive signs in elderly people. Arch Gerontol Geriatr 2011;52: 250-252.

Benatti A, Castiglione A, Trevisi P, Bovo R, Rosignoli M, Manara R, Martini A: Endocochlear inflammation in cochlear implant users: case report and literature review. Int J Pediatr Otorhinolaryngol 2013;77:885-893.

Boi R, Racca L, Cavallero A, Carpaneto V, Racca M, Dall'Acqua F, Ricchetti M, Santelli A, Odetti P: Hearing loss and depressive symptoms in elderly patients. Geriatr Gerontol Int 2012;12:440-445.

Bovo R, Ciorba A, Martini A: Tinnitus and cochlear implants. Auris Nasus Larynx 2011a;38: 14-20.

Bovo R, Ciorba A, Trevisi P, Aimoni C, Cappiello L, Castiglione A, Govoni M, Martini A: Cochlear implant in Cogan syndrome. Acta Otolaryngol 2011b;131:494-497.

Castiglione A, Benatti A, Girasoli L, Caserta E, Montino S, Pagliaro M, Bovo R, Martini A: Cochlear implantation outcomes in older adults. Hearing Balance Commun 2015;13:86-88.
Chung J, Chueng K, Shipp D, Friesen L, Chen JM, Nedzelski JM, Lin VY: Unilateral multi-channel cochlear implantation results in significant improvement in quality of life. Otol Neurotol 2012;33:566-571.

Ciorba A, Bovo R, Trevisi P, Rosignoli M, Aimoni C, Castiglione A, Martini A: Postoperative complications in cochlear implants: a retrospective analysis of 438 consecutive cases. Eur Arch Otorhinolaryngol 2012;269:1599-1603.

Drewnowski A, Murdock BB Jr: The role of auditory features in memory span for words. J Exp Psychol Hum Learn Mem 1980;6:319-332.

Fattahi F, Geshani A, Jafari Z, Jalaie S, Salman Mahini M: Auditory memory function in expert chess players. Med J Islam Repub Iran 2015;29:275.

Francis HW, Yeagle JA, Thompson CB: Clinical and psychosocial risk factors of hearing outcome in older adults with cochlear implants. Laryngoscope 2015;125:695-702.

Kumar A, Foster TC: Neurophysiology of old neurons and synapses; in Riddle DR (ed): Brain Aging: Models, Methods, and Mechanisms. Frontiers in Neuroscience. Boca Raton, CRC Press, 2007.
Lazard DS, Giraud AL, Truy E, Lee HJ: Evolution of non-speech sound memory in postlingual deafness: implications for cochlear implant rehabilitation. Neuropsychologia 2011;49: 2475-2482.

Lazard DS, Lee HJ, Gaebler M, Kell CA, Truy E, Giraud AL: Phonological processing in postlingual deafness and cochlear implant outcome. Neuroimage 2010;49:3443-3451.

Lazard DS, Lee HJ, Truy E, Giraud AL: Bilateral reorganization of posterior temporal cortices in post-lingual deafness and its relation to $\mathrm{co}$ chlear implant outcome. Hum Brain Mapp 2013;34:1208-1219.

Lesher EL, Berryhill JS: Validation of the Geriatric Depression Scale-Short Form among inpatients. J Clin Psychol 1994;50:256-260.

Leung JL, Lee GT, Lam YH, Chan RC, Wu JY: The use of the digit span test in screening for cognitive impairment in acute medical inpatients. Int Psychogeriatr 2011;23:1569-1574.

Lin FR: Hearing loss and cognition among older adults in the United States. J Gerontol A Biol Sci Med Sci 2011;66:1131-1136. 
Lin FR, Ferrucci L, An Y, Goh JO, Doshi J, Metter EJ, Davatzikos C, Kraut MA, Resnick SM: Association of hearing impairment with brain volume changes in older adults. Neuroimage 2014;90:84-92.

Lin FR, Metter EJ, O'Brien RJ, Resnick SM, Zonderman $A B$, Ferrucci L: Hearing loss and incident dementia. Arch Neurol 2011a;68:214220.

Lin FR, Thorpe R, Gordon-Salant S, Ferrucci L: Hearing loss prevalence and risk factors among older adults in the United States. J Gerontol A Biol Sci Med Sci 2011b;66:582590.

Lin FR, Yaffe K, Xia J, Xue QL, Harris TB, Purchase-Helzner E, Satterfield S, Ayonayon HN, Ferrucci L, Simonsick EM: Health ABCSG: hearing loss and cognitive decline in older adults. JAMA Intern Med 2013;173:293-299.

Mackin RS, Ayalon L, Feliciano L, Arean PA: The sensitivity and specificity of cognitive screening instruments to detect cognitive impairment in older adults with severe psychiatric illness. J Geriatr Psychiatry Neurol 2010;23: 94-99.

Martini A, Bovo R, Trevisi P, Forli F, Berrettini S: Cochlear implant in children: rationale, indications and cost/efficacy (in Italian). Minerva Pediatrica 2013;65:325-339.

Martini A, Castiglione A, Bovo R, Vallesi A, Gabelli C: Aging, cognitive load, dementia and hearing loss. Audiol Neurootol 2014; 19(suppl 1):2-5.

Mosnier I, Bebear JP, Marx M, Fraysse B, Truy E, Lina-Granade G, Mondain M, Sterkers-Artieres F, Bordure P, Robier A, Godey B, Meyer B, Frachet B, Poncet-Wallet C, Bouccara D, Sterkers O: Improvement of cognitive function after cochlear implantation in elderly pa- tients. JAMA Otolaryngol Head Neck Surg 2015;141:442-450.

Muangpaisan W, Intalapaporn S, Assantachai P: Digit span and verbal fluency tests in patients with mild cognitive impairment and normal subjects in Thai community. J Med Assoc Thailand Chotmaihet Thangphaet 2010;93: 224-230.

Nasreddine ZS, Phillips NA, Bedirian V, Charbonneau S, Whitehead V, Collin I, Cummings JL, Chertkow H: The Montreal Cognitive Assessment, MoCA: a brief screening tool for mild cognitive impairment. J Am Geriatr Soc 2005;53:695-699.

Poissant SF, Beaudoin F, Huang J, Brodsky J, Lee DJ: Impact of cochlear implantation on speech understanding, depression, and loneliness in the elderly. J Otolaryngol Head Neck Surg 2008;37:488-494.

Prince M, Bryce R, Albanese E, Wimo A, Ribeiro W, Ferri CP: The global prevalence of dementia: a systematic review and metaanalysis. Alzheimers Dement 2013;9:63-75.

Rivera D, Perrin PB, Stevens LF, Garza MT, Weil C, Saracho CP, Rodriguez W, RodriguezAgudelo Y, Rabago B, Weiler G, Garcia de la Cadena C, Longoni M, Martinez C, OcampoBarba N, Aliaga A, Galarza-Del-Angel J, Guerra A, Esenarro L, Arango-Lasprilla JC: Stroop color-word interference test: normative data for the Latin American Spanish speaking adult population. Neurorehabilitation 2015;37:591-624.

Ryugo DK, Kretzmer EA, Niparko JK: Restoration of auditory nerve synapses in cats by cochlear implants. Science 2005;310:14901492.

Schroeder RW, Twumasi-Ankrah P, Baade LE, Marshall PS: Reliable digit span: a systematic review and cross-validation study. Assessment 2012;19:21-30.

Seo EH, Lee DY, Choo IH, Kim SG, Kim KW, Youn JC, Jhoo JH, Woo JI: Normative study of the Stroop color and word test in an educationally diverse elderly population. Int J Geriatr Psychiatry 2008;23:1020-1027.

Sheikh JI, Yesavage JA: Geriatric Depression Scale (GDS). Recent evidence and development of a shorter version; in Brink TL (ed): Clinical Gerontology: A Guide to Assessment and Intervention. New York, Haworth Press, 1986, pp 165-173.

Shin MS, Kim SK, Kim SS, Park MH, Kim CS, Oh $\mathrm{SH}$ : Comparison of cognitive function in deaf children between before and after cochlear implant. Ear Hear 2007;28:22S-28S.

Stroop JR: Studies of interference in serial verbal reactions. J Exp Psychol 1935;18:643-662.

Sung JE, Kim JH, Jeong JH, Kang H: Working memory capacity and its relation to Stroop interference and facilitation effects in individuals with mild cognitive impairment. Am J Speech Lang Pathol 2012;21:S166-S178.

Van Bavel J: The world population explosion: causes, backgrounds and projections for the future. Facts Views Vis Obgyn 2013;5:281-291.

Watkins MJ, LeCompte DC, Elliott MN, Fish SB: Short-term memory for the timing of auditory and visual signals. J Exp Psychol Learning Mem Cogn 1992;18:931-937.

Wong LLN, Yu JKY, Chan SS, Tong MCF: Screening of cognitive function and hearing impairment in older adults: a preliminary study. Biomed Res Int 2014;2014:1-7.

Wong PC, Jin JX, Gunasekera GM, Abel R, Lee ER, Dhar S: Aging and cortical mechanisms of speech perception in noise. Neuropsychologia 2009;47:693-703. 